\title{
Electric Cell-Substrate Impedance Sensing of Cellular Effects under Hypoxic Conditions and Carbonic Anhydrase Inhibition
}

\author{
Luciana Stanica, ${ }^{1,2}$ Mihnea Rosu-Hamzescu, ${ }^{1,2}$ Mihaela Gheorghiu, ${ }^{1}$ Miruna Stan, ${ }^{3}$ \\ Loredana Antonescu, ${ }^{1}$ Cristina Polonschii, ${ }^{1}$ and Eugen Gheorghiu ${ }^{1,2}$ \\ ${ }^{1}$ International Centre of Biodynamics, Bucharest, Romania \\ ${ }^{2}$ Faculty of Biology, University of Bucharest, Bucharest, Romania \\ ${ }^{3}$ Department of Biochemistry and Molecular Biology, Faculty of Biology, University of Bucharest, Bucharest, Romania \\ Correspondence should be addressed to Eugen Gheorghiu; egheorghiu@biodyn.ro
}

Received 7 July 2017; Revised 23 October 2017; Accepted 16 November 2017; Published 27 December 2017

Academic Editor: Hana Vaisocherova

Copyright ( 2017 Luciana Stanica et al. This is an open access article distributed under the Creative Commons Attribution License, which permits unrestricted use, distribution, and reproduction in any medium, provided the original work is properly cited.

\begin{abstract}
Tumor hypoxia provides a dynamic environment for the cancer cells to thrive and metastasize. Evaluation of cell growth, cell-cell, and cell surface interactions in hypoxic conditions is therefore highly needed in the establishment of treatment options. Electric cell-substrate impedance sensing (ECIS) has been traditionally used in the evaluation of cellular platforms as a real-time, labelfree impedance-based method to study the activities of cells grown in tissue cultures, but its application for hypoxic environments is seldom reported. We present real-time evaluation of hypoxia-induced bioeffects with a focus on hypoxic $\mathrm{pH}$ regulation of tumor environment. To this end, multiparametric real-time bioanalytical platform using electrical impedance spectroscopy (EIS) and human colon cancer HT-29 cells is advanced. A time series of EIS data enables monitoring with high temporal resolution the alterations occurring within the cell layer, especially at the cell-substrate level. We reveal the dynamic changes of cellular processes during hypoxic conditions and in response to application of acetazolamide (AZA), a carbonic anhydrase inhibitor. Optical evaluation and $\mathrm{pH}$ assessment complemented the electrical analysis towards establishing a pattern of cellular changes. The proposed bioanalytical platform indicates wide applicability towards evaluation of bioeffects of hypoxia at cellular level.
\end{abstract}

\section{Introduction}

Hypoxic environments are characteristic for fast growing, solid tumors [1]. The carefully orchestrated tumor cell response to hypoxia provides cancerous cells both a metabolic advantage and the capability to metastasize [2], hypoxia being associated with aggressive tumor proliferation, resistance to therapy, and a poor prognosis [3].

The reprogramming of metabolism towards anaerobic glycolysis, the typical attribute of hypoxic tumor cells, is responsible for the acidic milieu within the tumor microenvironment [4] that impacts on cancer development and treatment outcome. Nevertheless, metabolic profiles of glycolysis-impaired tumors (demonstrating that local glucose and oxygen availabilities independently affect tumor acidity [5]) have revealed that $\mathrm{CO}_{2}$ in addition to lactic acid was a significant source of acidity in tumors, indicating carbonic anhydrases (CAs) as important contributors in the pharmacological management of tumor development [5]. Whereas there are 16 types of CA isozymes (among them-CAI, II, III, VII, and XIII being cytoplasmic, CAIV, IX, XII, XIV, and XV-membrane-bound, $\mathrm{CAVa}-$ mitochondrial and $\mathrm{Vb}$ and CAVI, secreted), only the hypoxia-inducible CA isozymes, CAIX and CAXII, are known to promote tumor growth through regulating the $\mathrm{pH}$ in the tumor microenvironment [6] and to be tumor associated. Accordingly, there is a tremendous interest in designing and testing of targeted, potent CA inhibitors. As such, the list of the potential CA inhibitors is continuously expanded, with particular focus for the CAIX isoform due 
to its important role in regulating both extra- and intercellular $\mathrm{pH}$ towards tumor survival and growth [7], its highly active extracellular enzyme domain, and its hypoxiainduced expression that correlates with poor prognosis.

Acetazolamide (AZA), a heterocyclic primary sulfonamide, is a well-known inhibitor of the catalytic activity of CAs [6]. AZA is used to treat glaucoma, edema, heart failure, and mountain sickness, is reported to significantly reduce cell proliferation and induce apoptosis in hypoxic human cancer cells, and is therefore an important model inhibitor in the design of novel compounds. Often, the efficiency of CA inhibition is predicted based on cell-free assessment of enzymatic activity and endpoint biochemical cell analyses, information that is not suitable for guiding the tailored design of new inhibitors.

In contrast to the endpoint analyses, the use of label-free bioanalytical platforms allows dynamic evaluation of discrete, nonlethal effects of external stimuli at cellular level [8] and enables a more nuanced, multiparametric assessment of potential bioeffects [9]. These include changes in cell growth and morphology [10], cell locomotion, cell-cell communication [11], and other behaviors directed by the cell's cytoskeleton [12] under specific (including hypoxic) conditions. As such, they are invaluable tools for the development and preclinical testing of novel therapeutic compounds.

Bioelectrical impedance cell culture platforms have shown a broad utility in biomedical applications and were recently shown to provide momentum to cancer research [13]. At the core of these platforms is electrical impedance spectroscopy (EIS), a sensitive method that provides noninvasive and real-time detection of electrical and morphological parameters of cellular monolayers in response to extracellular stressors. EIS uses an alternating current (AC) of low amplitude, within a wide frequency range, to measure the impedance of the cells grown on electrodes. Depending on the morphology [14] (changes in cell shape and volume), degree of attachment (changes in cell-substrate adherence), and electrical properties, the cells that are on or between the electrodes influence the flow of the current, shaping the characteristics of the measured signal corresponding to selected frequencies [15]. As such, EIS enables the study of cell properties like attachment [16] and spreading [17], growth and proliferation $[18,19]$, micromotion [20], apoptosis [21], metastatic potential, and endothelial barrier functions and cell-cell interactions [22], being a powerful complement to multielectrode array (MEA) recording technologies [23]. Chemical, biological, or physical stimuli change the current pathways through and around the cell modifying the impedance of the system making EIS a sensitive enough method to be used to evaluate cellular responses to external stimuli [24-27] and cell behavior changes in response to external/environmental conditions, such as $\mathrm{pH}$, temperature, light, and chemical/biological substances. It is amenable for multiplexing, and it can be combined with optical analyses and microfluidics and controlled environmental conditions. Nevertheless, EIS cell analysis under hypoxic conditions is seldom reported [28-30].

Aiming to characterize the dynamics of cellular changes under hypoxic conditions, this study advances real-time evaluation of hypoxia-induced bioeffects with a focus on hypoxic $\mathrm{pH}$ regulation of tumor environment. We chose AZA to probe the capability of impedance sensing as a realtime assay of the inhibitory effect without influence from potential variations given by specific mechanisms of action, CA type, or localization, in view of broader biosensing relevance. The proposed bioanalytical platform combines HT-29 cell cultures on gold film electrode arrays with multifrequency impedance measurements and hypoxic conditions. Together with optical microscopy and $\mathrm{pH}$ assay, the platform enables assessment of bioeffects of hypoxia providing insight into dynamic changes of cellular processes as cancer cells are exposed to low oxygen environments and CA inhibition as a clinically relevant target for novel cancer therapeutics specific for hypoxic tumors.

\section{Materials and Methods}

2.1. Cell Cultures. Colorectal carcinoma cells HT-29 cells (ATCC HTB38) were cultured in Dulbecco-modified Eagle's medium (DMEM) supplemented with $10 \%$ fetal bovine serum (FBS) and penicillin-streptomycin (100 IU/mL-0.1 mg/mL). Cells were seeded at a concentration of $4.5 \times 10^{5}$ cells $/ \mathrm{mL}$ on IBIDI ECIS (electric cell-substrate impedance sensing) culture ware ( 8 W10E PET) and cultured for up to 2 days in standard growth conditions. Upon reaching confluence, the cells were subjected within a (MCO-20AIC Sanyo, Japan) $5 \% \mathrm{CO}_{2}$ incubator to specific growth conditions with either normal $\left(21 \% \mathrm{O}_{2}\right)$ or low oxygen $\left(1 \% \mathrm{O}_{2}\right)$ concentrations. For reoxygenation, following hypoxic conditions, the medium was replaced with fresh medium and the cells were kept in normoxic conditions. The $\mathrm{pH}$ of the culture medium was measured at the beginning of the experiment and immediately at the end of each experimental procedure.

2.2. Hypoxic Conditions. Hypoxic conditions were acquired using a modular incubator chamber (MIC-101, BillupsRothenberg Inc.) inserted within the MCO-20AIC main incubator and equipped with a dedicated measurement electrode array mount for the IBIDI ECIS culture ware. The atmosphere in the chamber consisted of $94 \% \mathrm{~N}_{2}, 1 \% \mathrm{O}_{2}$, and $5 \% \mathrm{CO}_{2}$.

2.3. CA Inhibition. Acetazolamide ( $\geq 99 \%$, powder, SigmaAldrich, 222.2 Da), a pancarbonic anhydrase inhibitor, was dissolved in 20\% dimethylsulfoxide (DMSO, Sigma-Aldrich) in PBS to prepare a $10 \mathrm{mM}$ stock solution that was further diluted in DMEM to a final concentration of $100 \mu \mathrm{M}$, prior to experiment. $0.2 \%$ DMSO was used as carrier control.

2.4. Electrical Impedance Spectroscopy Measurements. Electrical impedance spectroscopy enables real-time monitoring of the interaction between cells and substrate together with the study of cellular processes [31]. A 4294A Precision Impedance Analyzer (Agilent, Japan) interfaced with a multiplexing module (8 channels), developed in-house, was used for recording (AC applied potential of $100 \mathrm{mV}$ amplitude with zero DC bias) a time series of impedance data $\left(Z^{*}(\omega)=\right.$ $|Z(\omega)|^{*} e^{i * \theta(\omega)}$ - polar form depending on modulus $|Z(\omega)|$ 
and phase $\theta(\omega)$, with $\omega$-the angular frequency) corresponding to $100 \mathrm{~Hz}-100 \mathrm{kHz}$ frequency range (100 frequency points with logarithmic distribution). Data were collected and processed using a custom-developed LabView interface and represented as the time series of either single frequency impedances or equivalent circuit parameters. Single frequency data (the equivalent resistance corresponding to the real part of the complex impedance at $10 \mathrm{kHz}$ ) were normalized using the formula $\left(\operatorname{Re}[Z(10 \mathrm{kHz}, t)]-\operatorname{Re}\left[Z^{*}(10 \mathrm{kHz}, 0)\right]\right) /$ $\operatorname{Re}\left[Z^{*}(10 \mathrm{kHz}, 0)\right]$ using the software package OriginPro 8.5 (OriginLab). The whole spectra of the complex impedance $Z^{*}(\mathrm{fr}, t)=\operatorname{Re}\left[Z^{*}(\mathrm{fr}, t)\right]-i \operatorname{Im}\left[Z^{*}(\mathrm{fr}, t)\right]$ were analysed and complex fitted with a simplified equivalent circuit (ZView, Solartron Analytical, UK) to derive time evolutions of specific circuit parameters as function of hypoxic environment and CA inhibition effect.

All values are expressed as mean \pm standard deviation (SD) for a minimum of 3 experiments.

2.5. Optical Microscopy Experiments. Bright field transmitted and reflected light microscopy have been used to evaluate label-free, live cells subjected to normoxic and hypoxic conditions. The microscopy set-up contains an AxioObzerver Z1 (Zeiss, Germany) platform, a 20x 0.4 NA objective (Zeiss, Germany), an ANDOR DU885 EMCCD camera, and an environmental control enclosure $\left(\mathrm{CO}_{2}\right.$ and temperature, OKOLab, Italy). For advanced analyses, cells were seeded at a concentration of $5 \times 10^{4}$ cells $/ \mathrm{mL}$ on Petri dishes with glass bottom (World Precision Instruments Inc.) and assessed with a 40x 0.95 NA objective during experiments the next day.

Actin cytoskeleton morphology was imaged via fluorescence imaging using cells fixed with $4 \%$ paraformaldehyde for $20 \mathrm{~min}$ and permeabilized with $0.1 \%$ Triton X-100-2\% bovine serum albumin solution (prepared in PBS) for $1 \mathrm{~h}$ at room temperature. Filamentous actin (F-actin) was labelled with $20 \mu \mathrm{g} / \mathrm{mL}$ phalloidin conjugated with fluorescein isothiocyanate (FITC; Sigma-Aldrich, Germany) for $1 \mathrm{~h}$ at room temperature. Nuclei were stained with $2 \mu \mathrm{g} / \mathrm{mL}$ 4',6-diamidino-2-phenylindole (DAPI; Invitrogen, USA) for $10 \mathrm{~min}$ at room temperature.

2.6. Cell Proliferation Assay. Cell proliferation was assessed using the In Vitro Toxicology Assay Kit Sulforhodamine B (Sigma-Aldrich, USA) according to the manufacturer's instructions. Cells were fixed by adding 50\% trichloroacetic acid to the growth media in a $1 / 4$ ratio $(v / v)$, incubated for $1 \mathrm{~h}$ at $4^{\circ} \mathrm{C}$, and stained for $20 \mathrm{~min}$ with $0.4 \%$ sulforhodamine B. Afterwards, the cells were rinsed quickly with $1 \%$ acetic acid and the incorporated dye was solubilized with a $10 \mathrm{mM}$ Tris solution. The absorbance was measured at $490 \mathrm{~nm}$ using a spectrophotometer (Thermo Scientific, USA), and the results were represented as percentage relative to control (normoxic cells).

\section{Results}

EIS measurements in a wide frequency range $(100 \mathrm{~Hz}-$ $100 \mathrm{kHz}$ ) were performed to monitor cell growth and to evaluate in real-time and noninvasively the cell monolayer response to hypoxic conditions and application of AZA.

Single frequency analysis of the impedance data led to the identification of the frequency at which interfacial effects and membrane processes have the highest influence on the measured impedance values. The selected frequency was $10 \mathrm{kHz}$, a frequency suitable for monitoring the adhesion process of cells on the electrodes [21] that showed characteristic changes at cell surface level for both cell growth and hypoxic $\mathrm{pH}$ regulation (Figure 1). We chose in the representation of the cell dynamics under various conditions the real part of the measured complex impedance at the given frequency (as given by $\operatorname{Re}[Z(\omega, t)]=|Z(\omega, t)| * \cos [\theta(\omega)]$ ) to comprise in our analysis both the possible modulus and phase changes occurring as function of cell evolution.

When cells attach and spread on the electrodes, the electrical impedance increases as the dielectric cell bodies impede current flow between the electrodes (Figure 1(a)). Accordingly, the evolution of the real part of impedance at $10 \mathrm{kHz}$ enables evaluation of cell monolayer establishment. After confluence was achieved and impedance values reached a plateau (Figure $1(\mathrm{a})$ ), after $\sim 48 \mathrm{~h}$, cells were exposed to hypoxic conditions.

Cell adaptation via specific responses evolved to protect the cell from permanent damage or death is ubiquitous [32]. Likewise, the hypoxic conditions trigger specific cell adaptation to low oxygen availability with a vast literature concerning HT-29 ability to withstand and adapt to hypoxic conditions [33-35]. The complexity of the signaling networks involved in cell response to hypoxia is overarching, the stabilization and activation of the transcription factor HIF-1 (usually degraded in normoxic conditions) and its downstream targets being a hallmark of cell response to hypoxic conditions. CAs (particularly CAIX) represent one of the HIF-1 targets related to glycolysis and $\mathrm{pH}$ regulation that also include monocarboxylate transporter, glucose transporter-1, lactate dehydrogenase, and pyruvate dehydrogenase kinase [36].

As shown in Figure 1(b), consistent with cell adaptation to hypoxic conditions, during the first two hours of hypoxia, the impedance values reveal a small transient drop corresponding to cell rearrangement, including detachment of some cells from the surface.

As cells start to adapt to the hypoxic environment, a biphasic behavior is evident: after an initial increase of $~ 5 \%$ of the normalized impedance values, after $6 \mathrm{~h}$ of hypoxia, the impedance values start to decrease (Figure 1(b), red line). To check if the recorded impedance changes are associated with cell surface changes or cell death, we monitored the subsequent $12 \mathrm{~h}$ of reoxygenation as well as an additional step of hypoxia. While prolongation of hypoxic conditions (hypoxia II) with an additional $12 \mathrm{~h}$ reveals massive electrode uncoverage, the hypoxic cells detaching from the surface, when the cells subjected to hypoxia were reexposed to normoxic oxygen conditions (reoxygenation), the impedance values (error bars should be taken into account) confirm that most of the cells were viable and managed to adapt to the hypoxic stress with subsequent structural and morphological changes. Aware of the stabilization and activation of HIF-1 as function 


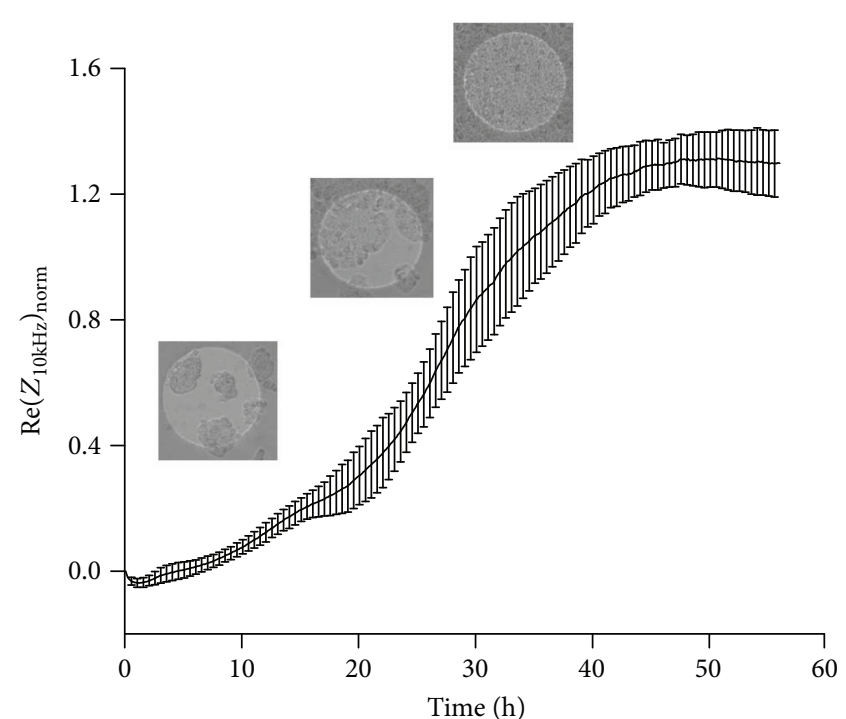

(a)

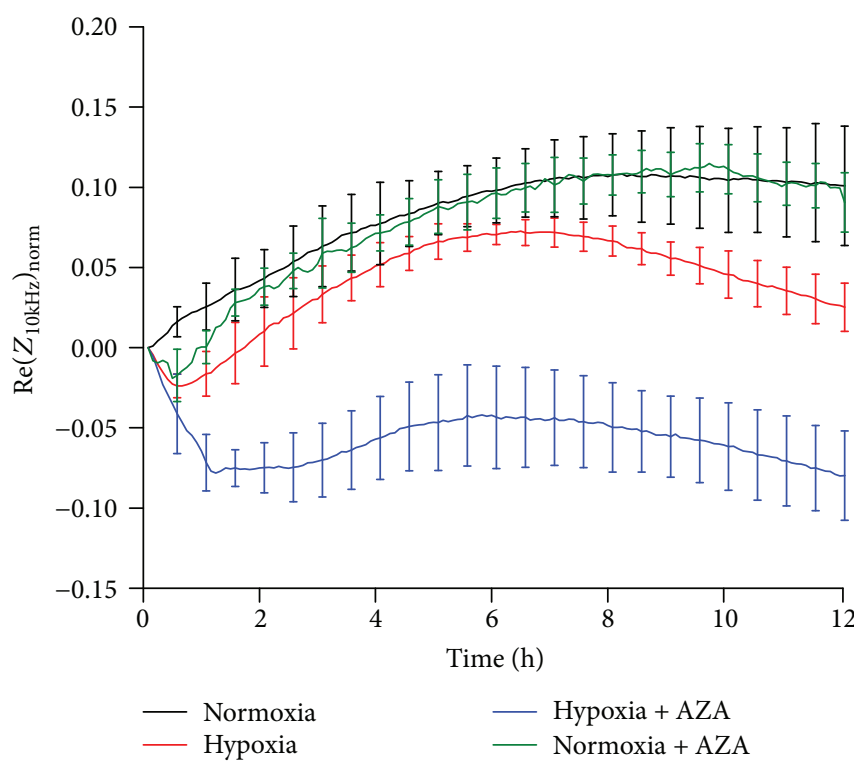

(c)

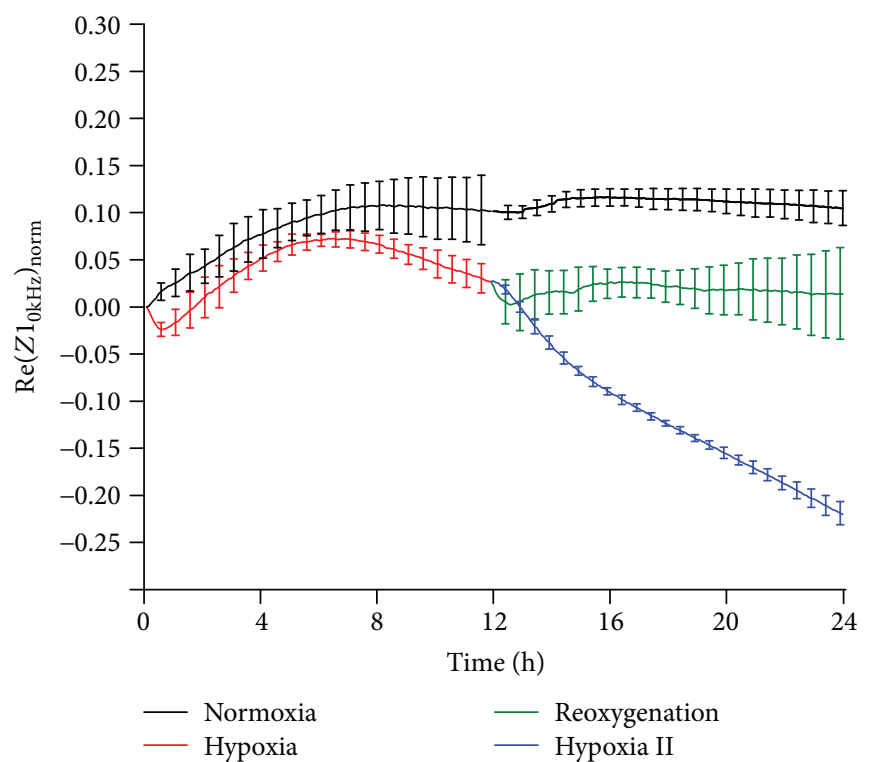

(b)

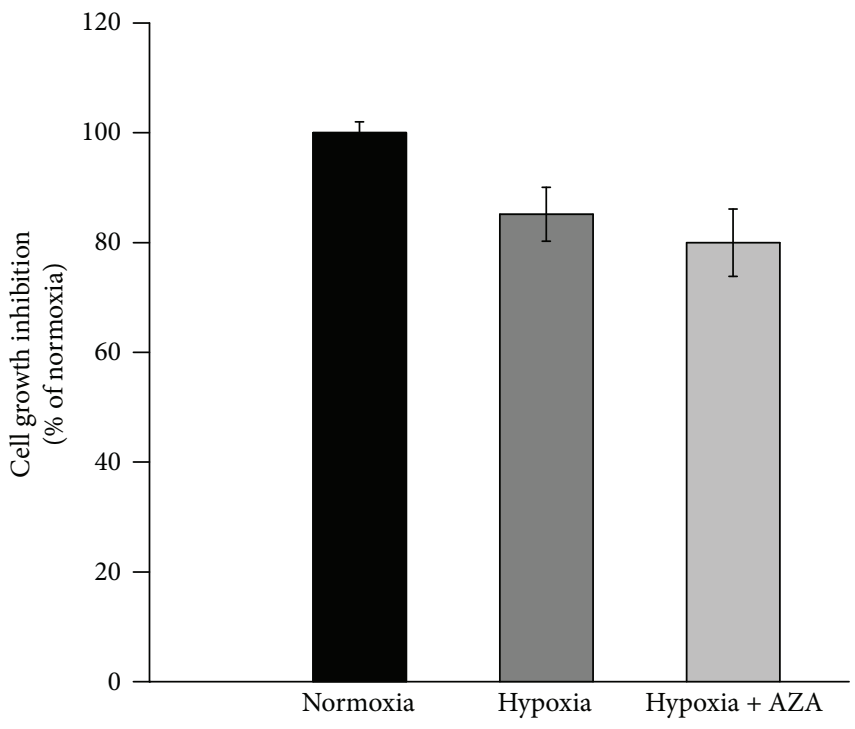

(d)

Figure 1: Impedance data, the real part of complex impedance at $10 \mathrm{kHz}$. (a) Cellular growth. Data are represented as mean \pm SD for $n=6$. (b) Cellular response to normoxia, hypoxia, reoxygenation, and a second stage of hypoxia. Data are represented as mean \pm SD for $n=6$ for normoxia, $n=8$ for hypoxia, $n=3$ for hypoxia II, and $n=5$ for reoxygenation. (c) Cellular dynamics under normoxia, normoxia and $100 \mu \mathrm{M}$ AZA, hypoxia alone, and hypoxia plus AZA. 0.2\% DMSO was used as vector control in all experiments. Data are represented as mean \pm SD for $n=5$ for normoxia, normoxia and AZA, hypoxia and AZA, and $n=8$ for hypoxia alone. (d) Complementary cell growth assay confirming moderate reduction of cell proliferation under hypoxic conditions $(<20 \%)$ and AZA inhibition. The results are represented as percentage of normoxia (control), considered $100 \%$. Data are expressed as mean \pm SD for $n=6$.

of cell density [37], cell confluency at the beginning of the experiment was controlled to be below $100 \%$.

From a molecular perspective, corresponding to the onset of hypoxic conditions, upregulation of CAs (and in particular of CAIX as result of transcriptional activation of the CA9 gene by HIF-1) [38] is commonly reported. Whereas CAIX is expressed (yet not catalytically active) in HT-29 cells even under normoxia, CAIX activation and overexpression are characteristic for hypoxic conditions [39]. Using the exact cell line and a fluorescent, specific CAIX inhibitor, we have recently [40] demonstrated that hypoxia triggered CAIX expression in HT-29 live cell experiments. After $8 \mathrm{~h}$ of 
hypoxia, we showed the specific fluorescent signal corresponding to active CAIX. Importantly, upon hypoxic conditions (as early as $30 \mathrm{~min}$ of hypoxia), the existing CAIX becomes catalytically active and was able to bind inhibitors (AZA included) [39].

Notably, CAIX is a functional mediator of both tumor growth and metastasis under hypoxic conditions [41]. As such, whereas impedance values are not specific to certain molecular processes, to test this adaptation, we used a known $\mathrm{CA}$ inhibitor, AZA, at $100 \mu \mathrm{M}$ concentration. The concentration was chosen below the therapeutic concentration shown to inhibit $60-70 \%$ of CA function [42]. In contrast to most of the available literature data that provide only endpoint analyses, our study is able to highlight the dynamics of cell layer adaptation under hypoxic conditions and upon mild CA inhibition.

These dynamic changes are exquisitely revealed using impedance: Figure 1(c) highlights the distinct evolutions for normoxic, normoxic plus CA inhibition, hypoxic, and hypoxic plus CA inhibition conditions. A monotonous $10 \%$ increase of impedance values is associated with monolayer tightening in normoxic conditions, whereas the biphasic dynamics revealed in hypoxic conditions are further altered due to AZA bioeffects. In comparison to hypoxia alone, the application of AZA reveals a further decrease of the impedance values indicating morphological changes of cells on the electrode surface, as cells' adaptation to hypoxic environment is impeded (Figure 1(c)) due to CA inhibition.

Complementary cell growth assay, sulforhodamine test (Figure 1(d)), shows moderate reduction of cell proliferation under hypoxic conditions (up to $20 \%$ change for hypoxic conditions and AZA exposure), in agreement with literature reports [34] where an $8 \mathrm{~h}$ exposure of human adenocarcinoma HT-29 cells to hypoxia resulted in $14 \%$ loss of viability, as assayed with trypan blue.

With appropriate frequency selection, single frequency analysis is able to reveal characteristic cell dynamics with sensing potential. Nevertheless, it is an over simplification of the complexity of the bioanalytical platform. To evaluate in real-time and noninvasively the cell monolayer response to hypoxic conditions and application of AZA, the experimental data obtained from the whole measured frequency domain were fitted with an equivalent electrical circuit whose elements, constant phase element (CPE), resistors, and capacitors are attributed to components in the biological probe such as paracellular space, cellular monolayer, or cell surface contacts. The circuit considered adequate to fit the measured impedance spectra is described by a constant phase element (CPE) which represents the cell monolayer-electrode interface, a resistor $\left(R_{\mathrm{p}}\right)$ that defines the paracellular route in parallel with a capacitor $\left(C_{\mathrm{c}}\right)$ which describes the cellular monolayer and a resistor for the bulk solution $\left(R_{\mathrm{sol}}\right)$ (Figure 2(a)). The impedance of CPE is characterized by two independent parameters, the amplitude, $\mathrm{CPE}_{-}$, and the distribution parameter, the exponent $\mathrm{CPE}_{-}$, according

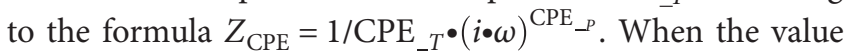
of $\mathrm{CPE}_{-P}$ equals 1 , the $\mathrm{CPE}$ behaves as an ideal capacitor, whereas when 0 , the $\mathrm{CPE}$ behaves as a pure resistor. $\mathrm{CPE}$ is used in the model, associated with the double-layer capacitance, to compensate for nonhomogeneity in the system in conjunction with the rough/porous surface of the electrodes and inhomogeneous cell attachment. Indeed, a constant phase element with a $\mathrm{CPE}_{P}$ value between 0.9 and 1 has been derived from the fitting.

Although the equivalent circuit is not unique, it was selected to enable long time assessment of impedance changes via complex fitting under different experimental conditions. A comparison between the experiment and fit for characteristic modulus and phase spectra is shown in Figure 2(b).

The normalized values of the elements of the electrical circuit obtained after fitting were compared $((V(t)-V(0)) /$ $V(0) \bullet 100)$ for normoxic cells, hypoxic cells, and hypoxic cells incubated with AZA to obtain complementary information regarding the bioeffects induced by these conditions on cells.

The most relevant changes were revealed in $R_{\mathrm{p}}$ and $C_{\mathrm{c}}$ values (Figures $3(\mathrm{a})$ and $3(\mathrm{~b})$ ) with modest changes of the $\mathrm{CPE}$ and $R_{\text {sol }}$ parameters (Figures 3(c) and 3(d)).

An increase of $R_{\mathrm{p}}$ for hypoxic cells corresponds to an increase in the monolayer tightness (cell-cell contacts) which occurs when cells begin to adapt to the low oxygenated environment and flatten on the surface. For the hypoxic cells incubated with AZA, the $R_{\mathrm{p}}$ decreases in accordance with a decrease of the cell surface adherence and cell-cell interaction (Figure 3(a)). The evolution of normalized $C_{c}$ values (inverted for better comparison: $\left.\left(C_{c}(0)-C_{c}(t)\right) / C_{c}(0) \bullet 100\right)$ for hypoxic cells is similar with the one in normoxic conditions, indicating a good coverage of the electrodes both upon adaptation to new low oxygen and for normal cell growth conditions, respectively. On the other hand, for hypoxic cells treated with AZA, $C_{c}$ changes, although modest $(10 \%)$, highlight a looser cell attachment and coverage of the electrodes (Figure 3(b)). These values corroborate with the values of the decreased $R_{\mathrm{p}}$ component pinpointing to morphological, cell-cell, and cell surface changes that are induced under combined hypoxic and AZA exposure.

As demonstrated in complementary biochemical evaluation of cell proliferation (Figure 1(d)), hypoxic conditions are able to induce a decrease in cell number. This reduction and associated changes in cell attachment to the surface can be related to the observed drift in CPE $(<2 \%)$ values (Figure 3(c)).

The drift in $R_{\text {sol }}$ parameters $(<5 \%$ Figure $3(\mathrm{~d}))$ has a similar trend for all experimental conditions and can be related to cell metabolism-induced changes in batch cultivation conditions.

To validate the impedance findings and shed light on the cellular changes responsible for these characteristic trends, advanced microscopy (Figure 4) has been deployed on both live and fixed cells. Differential interference contrast (DIC) and bright field reflected light (BFRL) images achieved in label free-conditions, on live cells, highlight the morphology of HT cells as a whole and the quality of the cell surface attachment, respectively. BFRL assay has been chosen for its ability to highlight cell areas not in full contact with the support surface. In the BFRL set-up, there will be a relatively strong reflection, and the background will be bright, for areas devoid of cells, while in the presence of a cell the background 


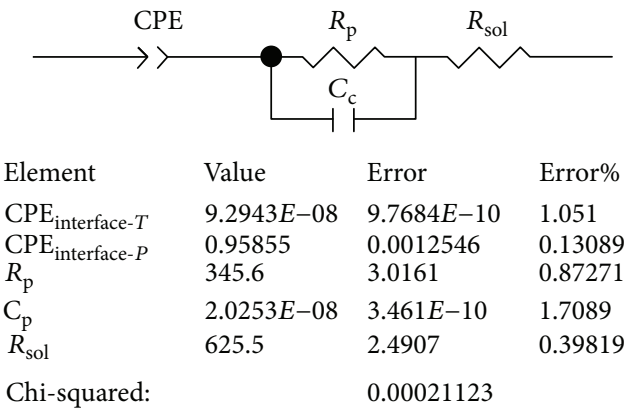

(a)

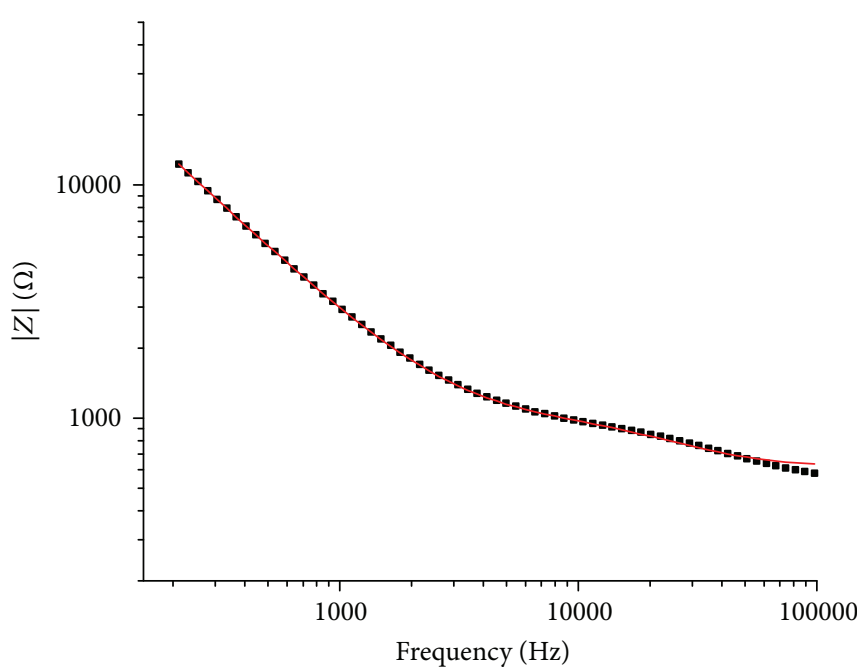

Experimental data - Fit result

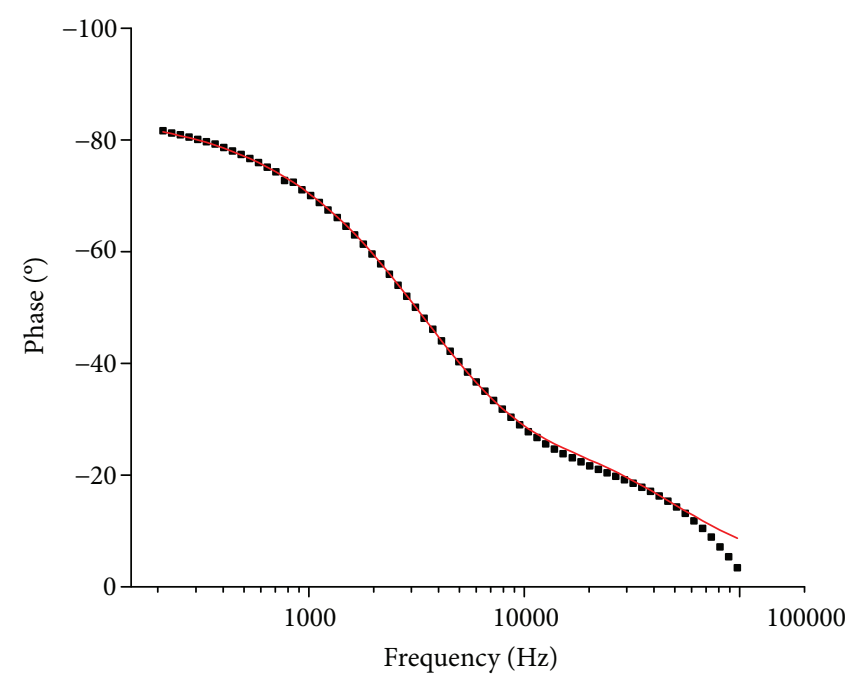

Experimental data - Fit result

(b)

FIgURE 2: (a) Simplified equivalent circuit for assessing EIS data on HT-29 cell monolayers: constant phase element (CPE) for the cell monolayer-electrode interface, $R_{\mathrm{p}}$ resistor that defines the paracellular route in parallel with a capacitor $\left(C_{\mathrm{c}}\right)$ which describes the cellular monolayer and a resistor for the bulk solution $\left(R_{\text {sol }}\right)$. (b) Complex impedance spectra, modulus, and phase, comparison between characteristic experimental values and fit.

intensity will be changed [43]. The different gray levels in the adhesion topology image correlate with the distance between the cell membrane and the glass interface (dark area = close contact area) [44]. A flatter morphology (evident in the DIC contrast) and more stress vacuoles (arrows in the DIC image) are revealed in DIC assay. Intriguingly, larger contacts have been recently reported for highly metastatic cells than for less metastatic ones using BFRL interference assays [45]. Our BFRL assays reveal for hypoxic cells exposed to AZA a more diffuse cytoskeleton (visible as wider reflecting areas-the white zones-in the centre of the cells) and possible cell integrity effects (arrows in BFRL images revealing areas of cell detachment or disorganization of cortical ring-shaped actin).

The fluorescence data, with cell nucleus labeled with DAPI and the F-actin within the cytoskeleton labelled with phalloidin-FITC, were assayed on fixed cells and reveal a more spread and diffuse cytoskeleton, highlighted as a wider green area surrounding cell nuclei, for hypoxia and mixed exposure (hypoxia + AZA) in comparison with control images. Moreover, areas where the cell integrity is affected, that is, areas where the cortical ring-shaped actin on cell borders disappears (arrows) are visible under mixed exposure (hypoxia + AZA) conditions, further confirming DIC and BFRL data.

Inability to control intracellular $\mathrm{pH}$ has adverse consequences for different cellular processes including proliferation and survival [46]. In response to hypoxia, cells adapt and reset their metabolism towards anaerobic glycolysis, responsible for the acidic milieu within the tumor microenvironment and express CAs (in particular CAIX which plays an important role in regulating both extra- and intercellular $\mathrm{pH}$ ) towards tumor survival and growth [47]. The hypoxiainduced CAs maintain the intracellular $\mathrm{pH}$ favorable for tumor cells while contributing to the acidification of the tumor environment and promoting tumor metastasis. To do so, CAs regulate $\mathrm{pH}$ and ion balance by catalysing the interconversion of carbon dioxide $\left(\mathrm{CO}_{2}\right)$ to bicarbonate anion $\left(\mathrm{HCO}_{3}{ }^{-}\right)$and protons $\left(\mathrm{H}^{+}\right)$[7]. To check the effect of hypoxic conditions and inhibitor exposure, samples of the growth medium at the beginning of the experimental assay and after $12 \mathrm{~h}$ of cell cultivation were assayed using standard 


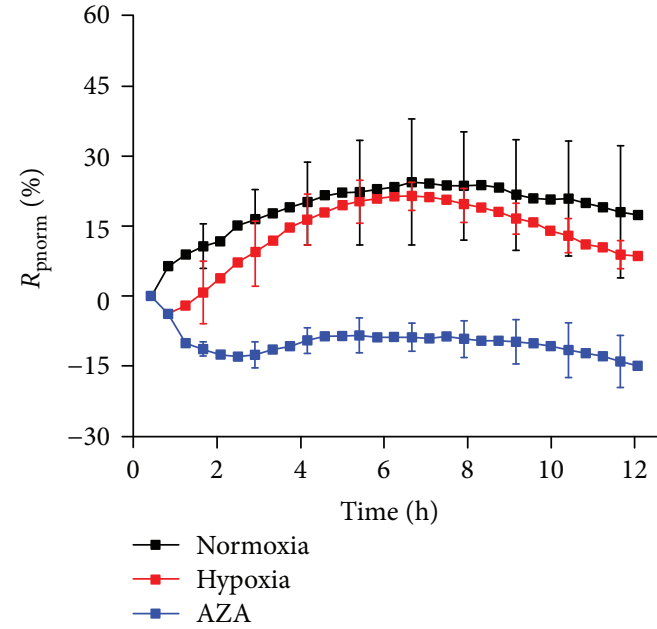

(a)

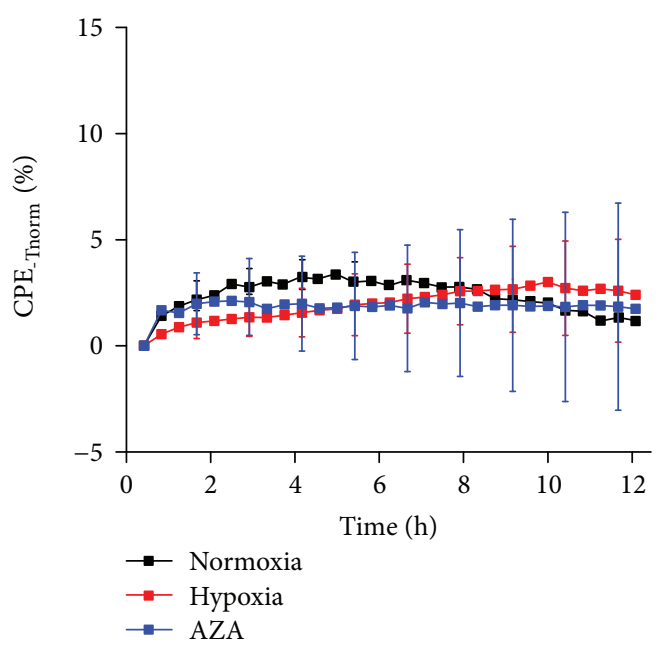

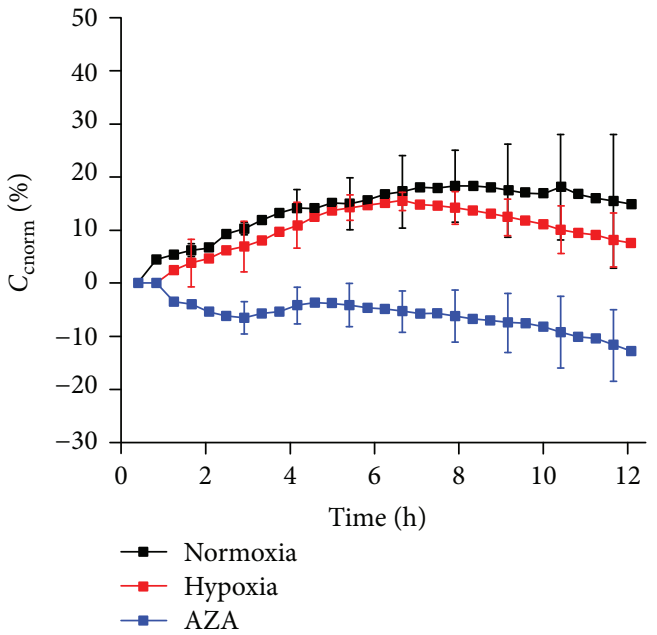

(b)

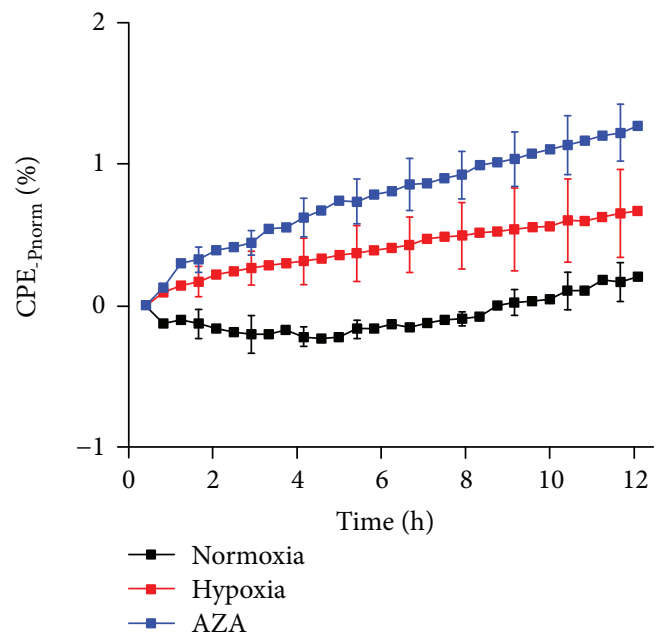

(c)

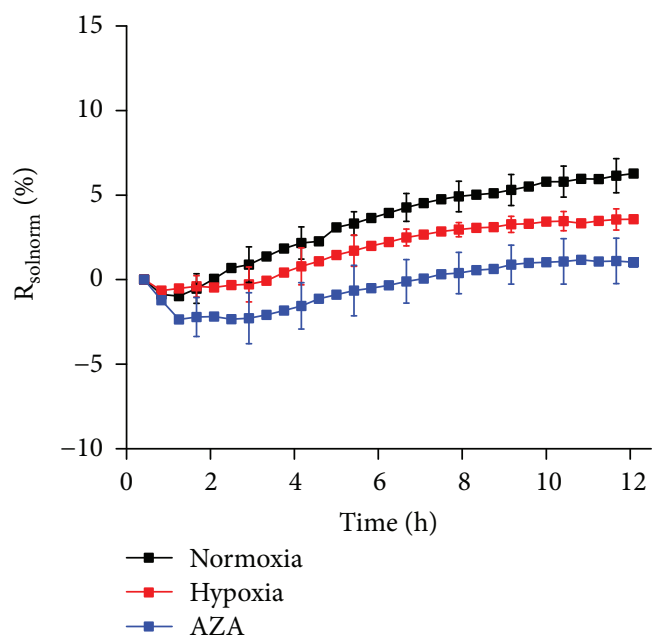

(d)

Figure 3: Evolution of cell monolayer electrical parameters derived based on fitting. (a) Paracellular resistance ( $\left.R_{\mathrm{p}}\right)$. (b) Cell monolayer capacitance $\left(C_{\mathrm{c}}\right)$. (c) Constant phase element $\left(\mathrm{CPE}_{-T}\right.$ and $\mathrm{CPE}_{P}$ parameters). (d) Bulk resistance $\left(R_{\text {sol }}\right)$. Data are represented as mean $\pm \operatorname{SD}$ for $n=3$. 


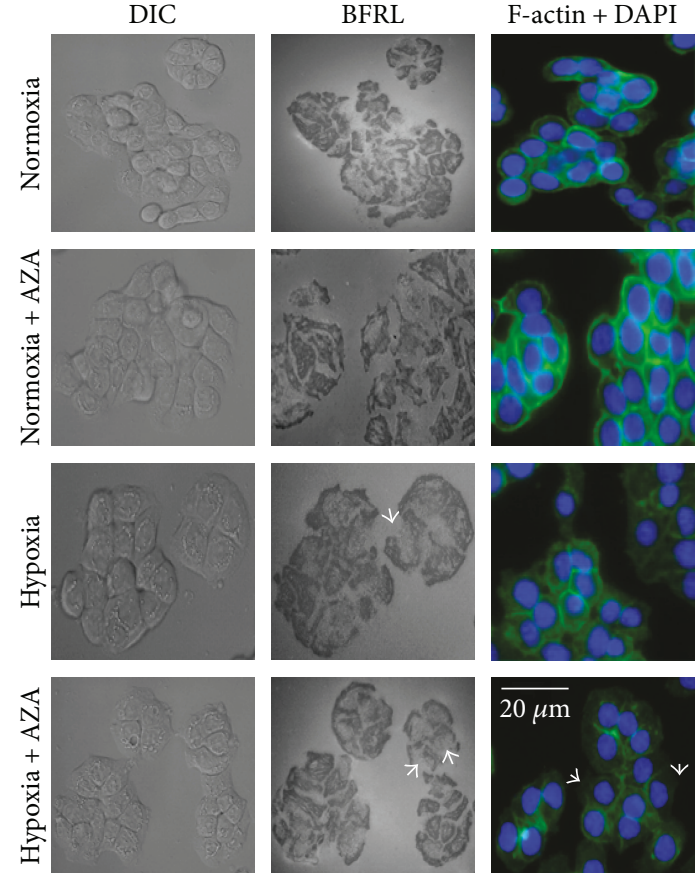

Figure 4: Microscopy images of normoxic and hypoxic cells (control) as well as exposed to AZA inhibitor. Differential interference contrast (DIC) images of live cells show a flatter morphology and more stress vacuoles (arrows) while bright field reflected light (BFRL) images show a more diffuse cytoskeleton for hypoxic cells; arrows in BFRL images reveal areas of cell detachment or disorganization of cortical ring-shaped actin. Fluorescence data (nucleus-DAPI and F-actin labelled with phalloidin-FITC), on fixed cells, reveal a more diffuse cytoskeleton for hypoxic cells and the disappearance of cortical ring-shaped actin (indicated by arrows). Scale bar, $20 \mu \mathrm{m}$.

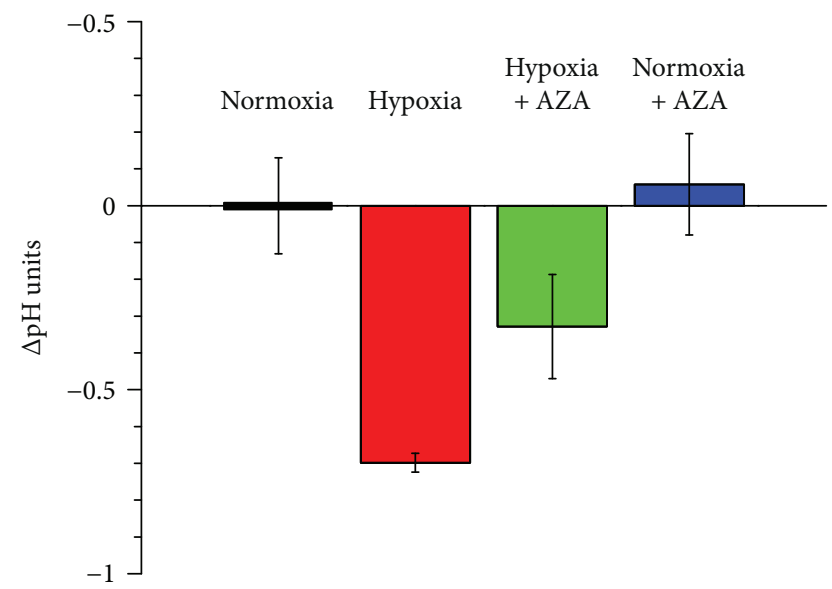

FIGURE 5: Extracellular $\mathrm{pH}$ changes after 12 hours of cell cultivation in different normoxic and hypoxic conditions. AZA was added just before incubation under normoxia or hypoxia, and the $\mathrm{pH}$ of the culture medium was measured from at least four independent experiments. $0.2 \%$ DMSO has been used as vector control in all experiments. Data are expressed as mean \pm SD for $n=4$ and represent the difference between $\mathrm{pH}$ values measured in normoxia, hypoxia, hypoxia with AZA, and normoxia with AZA and the initial $\mathrm{pH}$ values.
$\mathrm{pH}$ meter. $\mathrm{pH}$ evaluation indicated that indeed, hypoxic conditions led to a relative acidification of the extracellular cell culture medium (Figure 5). Following incubation with $100 \mu \mathrm{M}$ of AZA in hypoxic conditions, the decrease of the extracellular $\mathrm{pH}$ is reduced in accordance with inhibitory effect of AZA on the active CAs [7]. Moreover, the effect of AZA on the normoxic extracellular $\mathrm{pH}$ was negligible.

\section{Discussions}

Hypoxic environments are characteristic for fast growing, solid tumors, being associated with aggressive tumor proliferation, resistance to therapy, and a poor prognosis.

Impedance studies with cells in hypoxic conditions are seldom reported $[29,48]$, with a focus on barrier function assays. In contrast to the endpoint analyses, the use of this label-free bioanalytical platform allows dynamic evaluation of discrete, nonlethal effects of external stimuli at cellular level and enables a quantitative, multiparametric assessment of potential bioeffects. Our study demonstrates dynamic evaluation of cellular effects induced by hypoxic conditions and upon chemical inhibition of CAs and indicates a powerful biosensing approach for hypoxic cultures. The HT-29 cells were used as model cell lines for bioanalysis due to their good adherence, stable growth conditions, and ability to adapt to hypoxic conditions via overexpression of CAs (in particular CAIX considered an endogenous marker of hypoxia), coordinating the carefully orchestrated tumor cell response to hypoxia that provides cancerous cells both a metabolic advantage and the capability to metastasize [6]. This role in tumor progression and high incidence of metastasis is due to the transmembrane CA isoform capacity to maintain tumor intracellular $\mathrm{pH}$ homeostasis while acidifying the extracellular environment [7]. Another important aspect of CA functionality, the potential to decrease cell-cell adhesion by destabilization of E-cadherin links to cytoskeleton, may contribute to the acquisition of increased tumor aggressiveness [49]. Targeting CAs in general, the current study exploits the demonstrated EIS success as a label-free, realtime, and noninvasive method in applications concerning cell differentiation, cell spreading, cell motility, toxicology, cytotoxicity, and cell cycle progression $[15,31]$ towards a quantitative analysis approach for evaluation of hypoxic conditions and a potential inhibitor efficacy as anticancer compound. We chose AZA to probe the capability of impedance sensing as a real-time assay of the inhibitory effect without influence from potential variations given by specific mechanisms of action, CA type, or localization, in view of broader biosensing relevance. As recently demonstrated by our group in a complementary study [40], targeted specificity and in depth understanding of the interaction between CAIX and its inhibitors can be also achieved.

Corroborated with literature data demonstrating that the early $(<1 \mathrm{~h})$ response of HT-29 cell under hypoxic conditions is governed by activation of the existing CAIX followed by the CAIX overexpression [39], our single frequency analysis of the impedance data (real part at $10 \mathrm{kHz}$ ) provides qualitative discrimination between different cultivation conditions and is able to reveal dynamically the effect of CA activity 
(including overexpression) under hypoxic conditions [7] and inhibition (using $100 \mu \mathrm{M}$ concentration of AZA) on cell monolayers. Cell adhesion and cytoskeletal activity serve as valuable indicators [21] for understanding cancer cell behavior, such as proliferation, migration, and invasion [13], and offers cell response profiles related to changes in cellular dynamics as a result of the interaction of extracellular compounds with cells [50]. To further quantify these effects, complex nonlinear fitting of the impedance data in a wide frequency range demonstrated via the derived equivalent circuit parameter characteristic changes of the monolayer tightness $\left(R_{\mathrm{p}}\right)$ and cell morphology $\left(C_{\mathrm{c}}\right)$ for hypoxic and perturbed $\mathrm{pH}$ homeostasy under $\mathrm{CA}$ inhibition.

By evaluating cell morphology (DIC), cell surface contacts (BFRL), and the actin cytoskeleton (fluorescence), as well as the extracellular medium acidification ( $\mathrm{pH}$ measurements), we were able to confirm the cellular effects induced by hypoxic conditions and upon AZA inhibition of CA highlighted noninvasively and label free using impedance bioassay.

In conclusion, the study provides an efficient sensing platform for the evaluation of hypoxic conditions and CA inhibition as a developmental cell-based sensor of novel anticancer compounds targeting tumor hypoxia as a dynamic environment.

\section{Conflicts of Interest}

The authors declare that there is no conflict of interest regarding the publication of this article.

\section{Authors' Contributions}

Luciana Stanica and Mihnea Rosu-Hamzescu have equally contributed.

\section{Acknowledgments}

The financial support by grants of the Executive Agency for Higher Education, Research, Development and Innovation Funding (UEFISCDI), Romanian Ministry of National Education and Scientific Research, Project nos. PNII-IDPCCE-2011-2-0075, PN-III-P2-2.1-PED-2016-1137, is gratefully acknowledged. The technical support of Mrs. Florica Moranescu is gratefully acknowledged.

\section{References}

[1] P. Vaupel, "The role of hypoxia-induced factors in tumor progression," The Oncologist, vol. 9, Supplement 5, pp. 10-17, 2004.

[2] W. R. Wilson and M. P. Hay, "Targeting hypoxia in cancer therapy," Nature Reviews. Cancer, vol. 11, no. 6, pp. 393-410, 2011.

[3] A. Wouters, B. Pauwels, F. Lardon, and J. B. Vermorken, "Review: implications of in vitro research on the effect of radiotherapy and chemotherapy under hypoxic conditions," The Oncologist, vol. 12, no. 6, pp. 690-712, 2007.
[4] D. Neri and C. T. Supuran, "Interfering with $\mathrm{pH}$ regulation in tumours as a therapeutic strategy," Nature Reviews Drug Discovery, vol. 10, no. 10, pp. 767-777, 2011.

[5] G. Helmlinger, A. Sckell, M. Dellian, N. S. Forbes, and R. K. Jain, "Acid production in glycolysis-impaired tumors provides new insights into tumor metabolism," Clinical Cancer Research, vol. 8, no. 4, pp. 1284-1291, 2002.

[6] C. T. Supuran, "Structure and function of carbonic anhydrases," Biochemical Journal, vol. 473, no. 14, pp. 2023-2032, 2016.

[7] E. Švastová, A. Hulíková, M. Rafajová et al., "Hypoxia activates the capacity of tumor-associated carbonic anhydrase IX to acidify extracellular pH," FEBS Letters, vol. 577, no. 3, pp. 439-445, 2004.

[8] M. Gheorghiu, A. M. Enciu, B. O. Popescu, and E. Gheorghiu, "Functional and molecular characterization of the effect of amyloid- $\beta_{42}$ on an in vitro epithelial barrier model," Journal of Alzheimer's Disease, vol. 38, no. 4, pp. 787-798.

[9] M. Gheorghiu, S. David, C. Polonschii et al., "Label free sensing platform for amyloid fibrils effect on living cells," Biosensors \& Bioelectronics, vol. 52, pp. 89-97, 2014.

[10] E. Gheorghiu, "Characterizing cellular systems by means of dielectric spectroscopy," Bioelectromagnetics, vol. 17, no. 6, pp. 475-482, 1996.

[11] Q. Liu, C. Wu, H. Cai, N. Hu, J. Zhou, and P. Wang, "Cellbased biosensors and their application in biomedicine," Chemical Reviews, vol. 114, no. 12, pp. 6423-6461, 2014.

[12] S. Ramasamy, D. Bennet, and S. Kim, "Drug and bioactive molecule screening based on a bioelectrical impedance cell culture platform," International Journal of Nanomedicine, vol. 9, pp. 5789-5809, 2014.

[13] J. Hong, K. Kandasamy, M. Marimuthu, C. S. Choi, and S. Kim, "Electrical cell-substrate impedance sensing as a noninvasive tool for cancer cell study," Analyst, vol. 136, no. 2, pp. 237-245, 2011.

[14] E. Gheorghiu, "On the limits of ellipsoidal models when analyzing dielectric behavior of living cells. Emphasis on red blood cells," Annals of the New York Academy of Sciences, vol. 873, no. 1 ELECTRICAL BI, pp. 262-268, 1999.

[15] J. Wang, C. Wu, N. Hu, J. Zhou, L. Du, and P. Wang, "Microfabricated electrochemical cell-based biosensors for analysis of living cells in vitro," Biosensors, vol. 2, no. 4, pp. 127-170, 2012.

[16] F. Asphahani, M. Thein, O. Veiseh et al., "Influence of cell adhesion and spreading on impedance characteristics of cellbased sensors," Biosensors \& Bioelectronics, vol. 23, no. 8, pp. 1307-1313, 2008.

[17] J. Wegener, C. R. Keese, and I. Giaever, "Electric cell-substrate impedance sensing (ECIS) as a noninvasive means to monitor the kinetics of cell spreading to artificial surfaces," Experimental Cell Research, vol. 259, no. 1, pp. 158-166, 2000.

[18] L. Wang, L. Wang, H. Yin et al., "Real-time, label-free monitoring of the cell cycle with a cellular impedance sensing chip," Biosensors and Bioelectronics, vol. 25, no. 5, pp. 990-995, 2010.

[19] G. Park, C. K. Choi, A. E. English, and T. E. Sparer, "Electrical impedance measurements predict cellular transformation," Cell Biology International, vol. 33, no. 3, pp. 429-433, 2009.

[20] I. Giaever and C. R. Keese, "A morphological biosensor for mammalian cells,” Nature, vol. 366, no. 6455, pp. 591-592, 1993.

[21] S. Arndt, J. Seebach, K. Psathaki, H. J. Galla, and J. Wegener, "Bioelectrical impedance assay to monitor changes in cell 
shape during apoptosis," Biosensors and Bioelectronics, vol. 19, no. 6, pp. 583-594, 2004.

[22] E. Gheorghiu, C. Balut, and M. Gheorghiu, "Dielectric behaviour of gap junction connected cells: a microscopic approach," Physics in Medicine and Biology, vol. 47, no. 2, pp. 341-348, 2002.

[23] M. E. Spira and A. Hai, "Multi-electrode array technologies for neuroscience and cardiology," Nature Nanotechnology, vol. 8, no. 2, pp. 83-94, 2013.

[24] X. Zhang, F. Li, A. N. Nordin, J. Tarbell, and I. Voiculescu, "Toxicity studies using mammalian cells and impedance spectroscopy method," Sensing and Bio-Sensing Research, vol. 3, pp. 112-121, 2015.

[25] T. M. Curtis, J. Tabb, L. Romeo, S. J. Schwager, M. W. Widder, and W. H. van der Schalie, "Improved cell sensitivity and longevity in a rapid impedance-based toxicity sensor," Journal of Applied Toxicology, vol. 29, no. 5, pp. 374-380, 2009.

[26] J. H. Yeon and J.-K. Park, "Cytotoxicity test based on electrochemical impedance measurement of HepG2 cultured in microfabricated cell chip," Analytical Biochemistry, vol. 341, no. 2, pp. 308-315, 2005.

[27] K. B. Male, B. Lachance, S. Hrapovic, G. Sunahara, and J. H. T. Luong, "Assessment of cytotoxicity of quantum dots and gold nanoparticles using cell-based impedance spectroscopy," Analytical Chemistry, vol. 80, no. 14, pp. 5487-5493, 2008.

[28] S. K. Sharma, J. L. Lucitti, C. Nordman, J. P. Tinney, K. Tobita, and B. B. Keller, "Impact of hypoxia on early chick embryo growth and cardiovascular function," Pediatric Research, vol. 59, no. 1, pp. 116-120, 2006.

[29] A. Chanakira, D. Kir, R. A. Barke, S. M. Santilli, S. Ramakrishnan, and S. Roy, "Hypoxia differentially regulates arterial and venous smooth muscle cell migration," PLoS One, vol. 10, no. 9, article e0138587, 2015.

[30] T. B. Tran, C. Baek, and J. Min, "Electric cell-substrate impedance sensing (ECIS) with microelectrode arrays for investigation of cancer cell - fibroblasts interaction," PLoS One, vol. 11, no. 4, article e0153813, 2016.

[31] W. Gu and Y. Zhao, "Cellular electrical impedance spectroscopy: an emerging technology of microscale biosensors," Expert Review of Medical Devices, vol. 7, no. 6, pp. 767-779, 2010.

[32] A. J. Fornace Jr., D. W. Nebert, M. C. Hollander et al., "Mammalian genes coordinately regulated by growth arrest signals and DNA-damaging agents," Molecular and Cellular Biology, vol. 9, no. 10, pp. 4196-4203, 1989.

[33] S. Carlin, N. Khan, T. Ku, V. A. Longo, S. M. Larson, and P. M. Smith-Jones, "Molecular targeting of carbonic anhydrase IX in mice with hypoxic HT29 colorectal tumor xenografts," PLoS One, vol. 5, no. 5, article e10857, 2010.

[34] K. S. Yao, M. Clayton, and P. J. O'Dwyer, "Apoptosis in human adenocarcinoma HT29 cells induced by exposure to hypoxia," Journal of the National Cancer Institute, vol. 87, no. 2, pp. 117122, 1995.

[35] K. S. Yao, S. Xanthoudakis, T. Curran, and P. J. O'Dwyer, "Activation of AP-1 and of a nuclear redox factor, Ref-1, in the response of HT29 colon cancer cells to hypoxia," Molecular and Cellular Biology, vol. 14, no. 9, pp. 5997-6003, 1994.

[36] G. L. Semenza, "HIF-1 mediates metabolic responses to intratumoral hypoxia and oncogenic mutations," The Journal of Clinical Investigation, vol. 123, no. 9, pp. 3664-3671, 2013.

[37] F. Dayan, R. L. Bilton, J. Laferrière et al., "Activation of HIF- $1 \alpha$ in exponentially growing cells via hypoxic stimulation is independent of the Akt/mTOR pathway," Journal of Cellular Physiology, vol. 218, no. 1, pp. 167-174, 2009.

[38] C. C. Wykoff, N. J. Beasley, P. H. Watson et al., "Hypoxiainducible expression of tumor-associated carbonic anhydrases," Cancer Research, vol. 60, no. 24, pp. 7075-7083, 2000.

[39] L. Dubois, K. Douma, C. T. Supuran et al., "Imaging the hypoxia surrogate marker CA IX requires expression and catalytic activity for binding fluorescent sulfonamide inhibitors," Radiotherapy and Oncology, vol. 83, no. 3, pp. 367-373, 2007.

[40] L. Stanica, M. Gheorghiu, M. Stan et al., "Quantitative assessment of specific carbonic anhydrase inhibitors effect on hypoxic cells using electrical impedance assays," Journal of Enzyme Inhibition and Medicinal Chemistry, vol. 32, no. 1, pp. 1079-1090, 2017.

[41] P.C. McDonald and S. Dedhar, "Carbonic anhydrase IX (CAIX) as a mediator of hypoxia-induced stress response in cancer cells," Sub-Cellular Biochemistry, vol. 75, pp. 255-269, 2014.

[42] B. Bao, K. Groves, J. Zhang et al., "In vivo imaging and quantification of carbonic anhydrase IX expression as an endogenous biomarker of tumor hypoxia," PLoS One, vol. 7, no. 11, article e50860, 2012.

[43] H. Verschueren, "Interference reflection microscopy in cell biology: methodology and applications," Journal of Cell Science, vol. 75, pp. 279-301, 1985.

[44] K. Klein, T. Maier, V. C. Hirschfeld-Warneken, and J. P. Spatz, "Marker-free phenotyping of tumor cells by fractal analysis of reflection interference contrast microscopy images," Nano Letters, vol. 13, no. 11, pp. 5474-5479, 2013.

[45] T. Matsuzaki, K. Ito, K. Masuda et al., "Quantitative evaluation of cancer cell adhesion to self-assembled monolayer-patterned substrates by reflection interference contrast microscopy," The Journal of Physical Chemistry B, vol. 120, no. 7, pp. 1221-1227, 2016.

[46] S. K. Parks, J. Chiche, and J. Pouyssegur, "pH control mechanisms of tumor survival and growth," Journal of Cellular Physiology, vol. 226, no. 2, pp. 299-308, 2011.

[47] P. C. McDonald, S. C. Chafe, and S. Dedhar, "Overcoming hypoxia-mediated tumor progression: combinatorial approaches targeting $\mathrm{pH}$ regulation, angiogenesis and immune dysfunction," Frontiers in Cell and Developmental Biology, vol. 4, p. 27, 2016.

[48] K. Kangwantas, E. Pinteaux, and J. Penny, "The extracellular matrix protein laminin-10 promotes blood-brain barrier repair after hypoxia and inflammation in vitro," Journal of Neuroinflammation, vol. 13, no. 1, p. 25, 2016.

[49] O. Sedlakova, E. Svastova, M. Takacova, J. Kopacek, J. Pastorek, and S. Pastorekova, "Carbonic anhydrase IX, a hypoxia-induced catalytic component of the $\mathrm{pH}$ regulating machinery in tumors," Frontiers in Physiology, vol. 4, p. 400, 2014.

[50] Y. A. Abassi, B. Xi, W. Zhang et al., "Kinetic cell-based morphological screening: prediction of mechanism of compound action and off-target effects," Chemistry \& Biology, vol. 16, no. 7, pp. 712-723, 2009. 


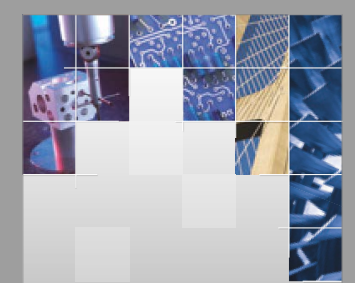

\section{Enfincering}
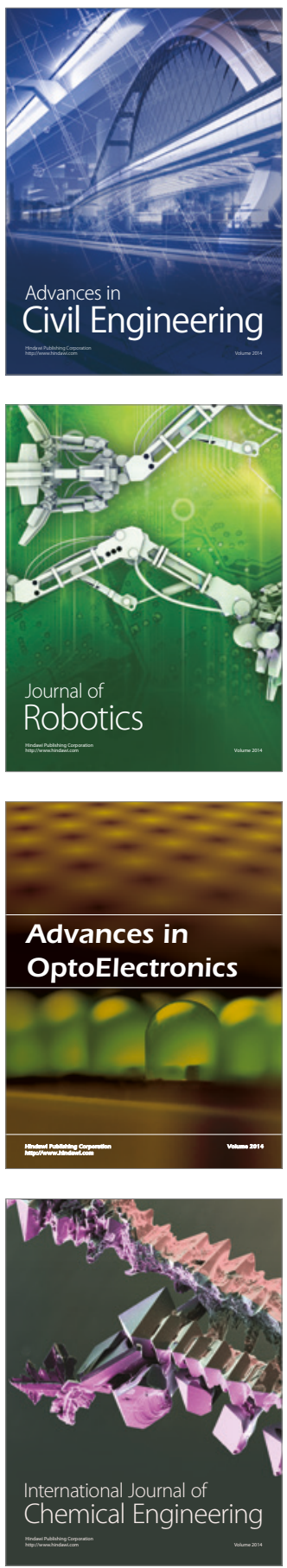

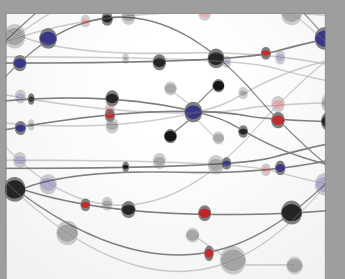

The Scientific World Journal

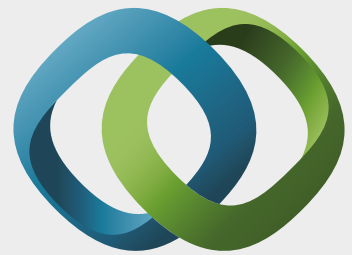

\section{Hindawi}

Submit your manuscripts at

https://www.hindawi.com
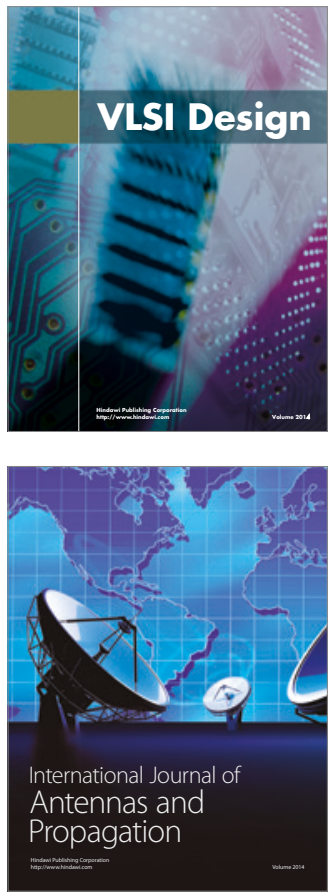

\section{Rotating}

Machinery
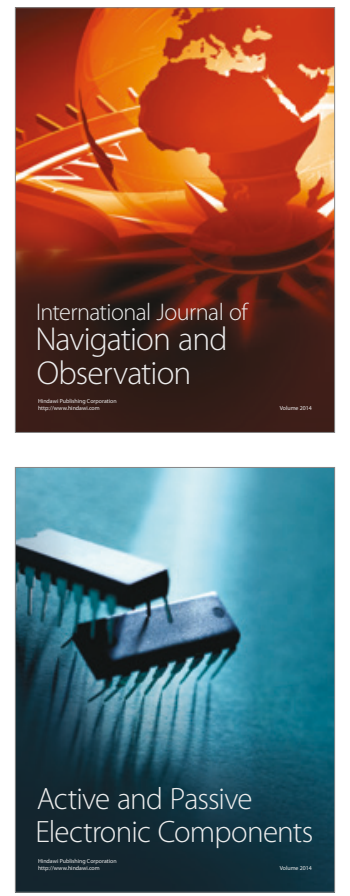
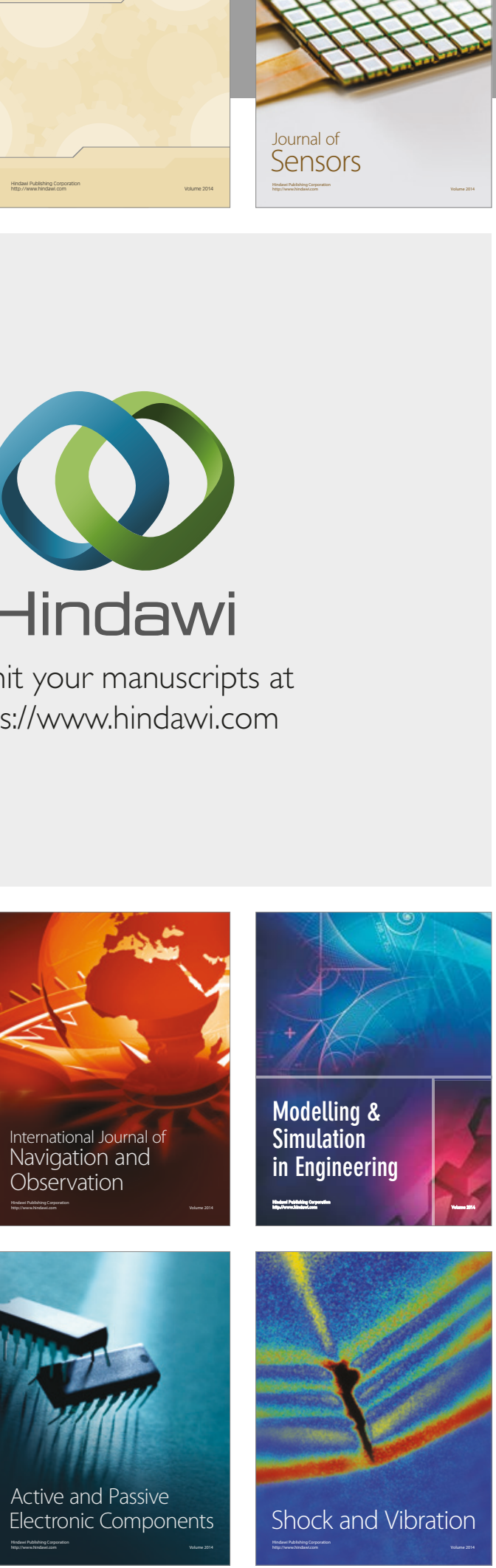
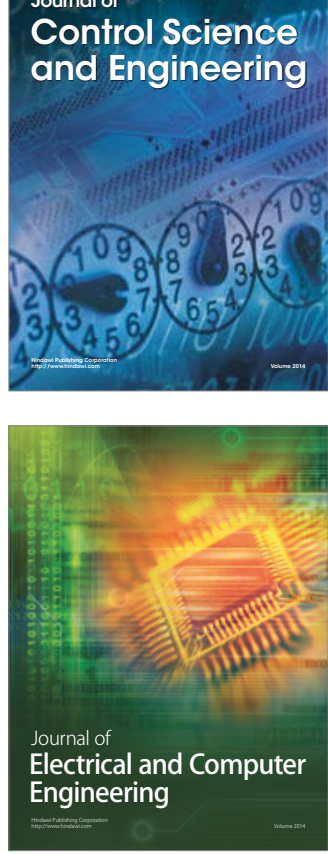

Distributed

Journal of

Control Science

and Engineering
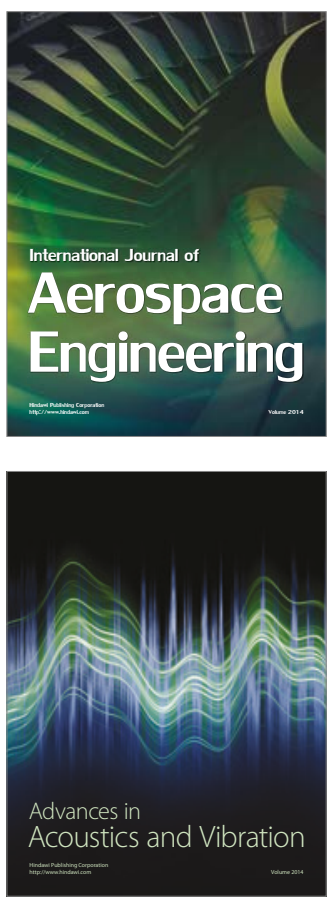

Sensor Networks 